\title{
Comparison and correlation of binding mode of ATP in the kinase domains of Hexokinase family
}

\author{
Yellapu Nanda Kumar1, Pasupuleti Santhosh Kumar², Gopal Sowjenya², Valasani Koteswara \\ Rao $^{3}$, Sthanikam Yeswanth', Uppu Venkateswara Prasad', Jangampalli Adi Pradeepkiran', \\ PVGK Sarma ${ }^{2} \&$ Matcha Bhaskar ${ }^{1 *}$
}

1Division of Animal Biotechnology, Department of Zoology, Sri Venkateswara University, Tirupati, Andhra Pradesh, India-517502; 2Department of Biotechnology, Sri Venkateswara Institute of Medical Sciences, Tirupati, AP, India-517507; 3Department of Pharmacology and Toxicology, University of Kansas, Lawrence, KS 66047, USA; Matcha Bhaskar - Email: matchabhaskar2010@gmail.com; Phone: +91-877-2289304 Ext: 111; Fax: +91-877-2289555; *Corresponding author

Received June 09, 2012; Accepted June 11, 2012; Published June 28, 2012

\begin{abstract}
:
Hexokinases (HKs) are the enzymes that catalyses the ATP dependent phosphorylation of Hexose sugars to Hexose-6-Phosphate (Hex-6-P). There exist four different forms of HKs namely HK-I, HK-II, HK-III and HK-IV and all of them share a common ATP binding site core surrounded by more variable sequence that determine substrate affinities. Although they share a common binding site but they differ in their kinetic functions, hence the present study is aimed to analyze the binding mode of ATP. The analysis revealed that the four ATP binding domains are showing 13 identical, 7 similar and 6 dissimilar residues with similar structural conformation. Molecular docking of ATP into the kinase domains using Molecular Operating Environment (MOE) soft ware tool clearly showed the variation in the binding mode of ATP with variable docking scores. This probably explains the variable phosphorylation rates among hexokinases family.
\end{abstract}

Key Words: Hexokinase, Molecular Docking, MOE

\section{Background:}

HK catalyzes the phosphorylation process by transferring the $\mathrm{y}$ phosphoryl group from ATP to the $\mathrm{OH}$ group of sixth carbon of Hexose sugars to give Hex-6-P. There are four isozymes of mammalian HKs namely HK-I, HK-II, HK-III and HK-IV, which are tissue specific and are located in different organs of the body [1, 2]. Liver contains all four types of HKs while kidney and intestine lacks HK-IV. HK-I and HK-II are found in epididymal fat pad, skeletal muscle, brain and heart. However, HK-I is predominantly present in brain and kidney and HK-II is predominant in skeletal muscle and epididymal fat pad [2]. The formation of Hex-6-P by HKs commits hexose sugars to alternative metabolic pathways: the formation of glycogen and short-term carbohydrate storage in liver, immediate use in energy production by glycolysis and the formation of pentose phosphates in the anabolic reactions [3] (Figure 1). Up regulation and down regulation of metabolic pathways can be linked to the different organs in the body and these differences may be attributed to the structure, affinity for substrates, inhibitors and sub cellular location of the isozymes [3]. HK-I and HK-II have a tail on the N-terminus that is important to bind with mitochondria whereas, HK-III and HK-IV lacks such structures and hence they are unable to bind to mitochondria. Thus, these isozymes may be associated with metabolic pathways other than glycolysis. All HKs share a common ATP binding site core surrounded by more variable sequence that determines substrate affinities. Although they share a common ATP binding site, the difference in their kinetic functions was observed [4]. This may be probably due to the variation in the active site residues and conformations which will finally affect 
the phosphorylation machinery. In order to ascertain these variations we carried out an insight structural analysis of all HKs concentrating on the kinase domain conformations. These different conformations may results in variable binding of ATP among HKs and hence there may be variation in the phosphorylation mechanism. In the present study we have carried out molecular docking study to predict the catalytic interactions between ATP and kinase domains of all HKs.

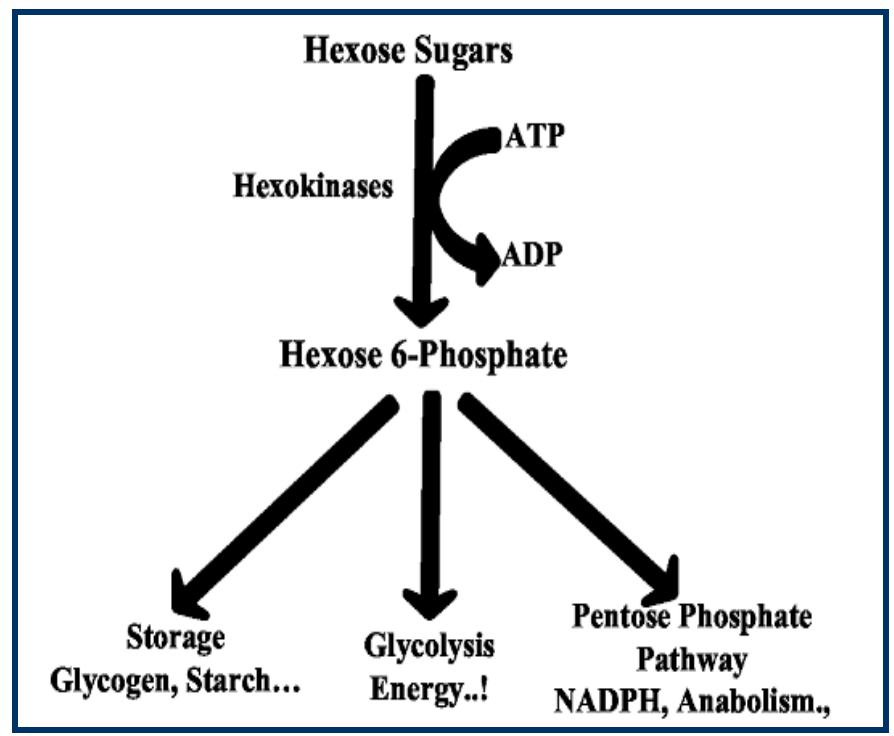

Figure 1: Fate of Hexose Sugars by Hexokinases

\section{Methodology:}

Hexokinase Structures

The three dimensional structures of HK-I (1HKC), HK-II (2NZT) and HK-IV (1V4S) were obtained from Protein Data Bank (PDB) [5]. As the structure of HK-III is not available so far in the PDB we have constructed its 3D model by homology modeling method.

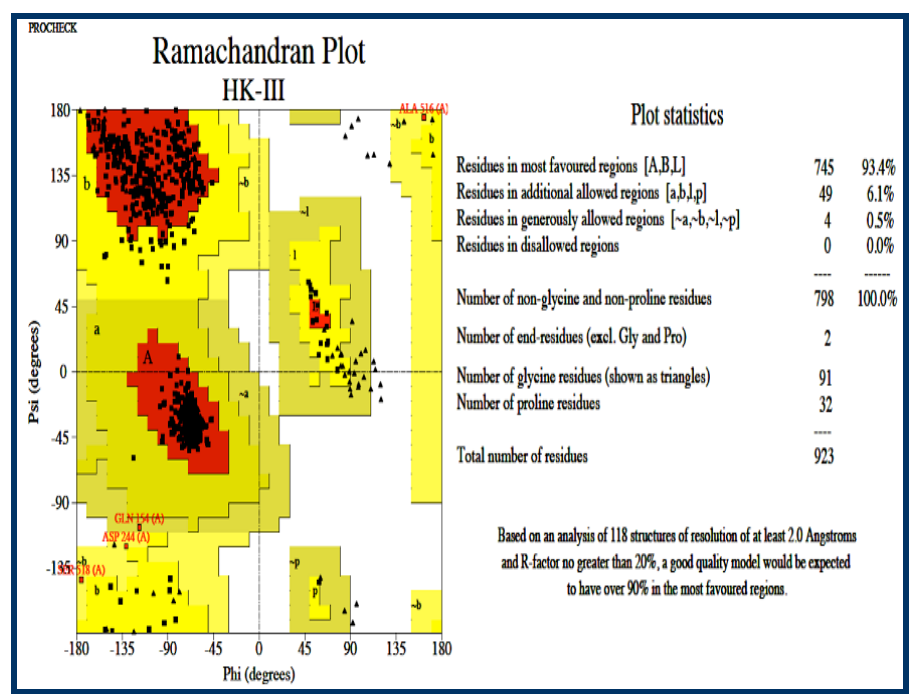

Figure 2: Ramachandran plot showing the stereo chemical quality of homology model of HK-III generated by PROCHECK validation server.

\section{Homology modeling of HK-III}

The three dimensional model of the HK-III was constructed by using Modeller 9v8 tool [6]. The HK-III protein sequence was retrieved from NCBI [7] (AC No: NP_002106.2) and it was subjected to BLASTp [8] against PDB and the crystal structure of human Hexokinase-II (PDB ID: 2NZT) was chosen as template for modeling which is having a maximum identity of $56 \%$. The protein sequence and 3D structure of the template were retrieved. A sequence alignment file was generated in PIR format for Query and template sequences using ClustalX tool [9], a Python script was written and 20 models were generated. Among 20, the model with the lowest DOPE score was selected for further analysis.

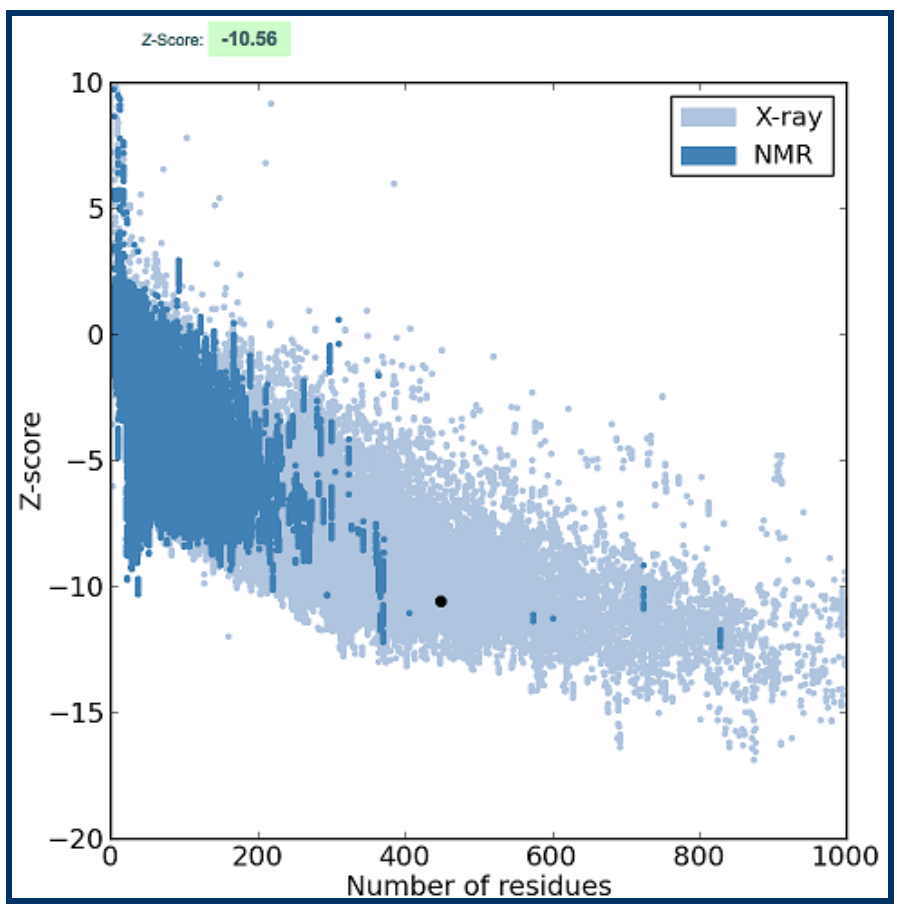

Figure 3: ProsaWeb quality plot of HK-III model showing a Zscore of -10.56 that is observed in the range of native X-Ray crystallographic structures.

\section{Validation of HK-III Model}

The stereo chemical quality of energy minimized HK-III model was assessed and validated by PROCHECK validation server [10]. The recognition of errors in this theoretical protein model is also a critical point employed in protein structure validation. Hence the overall quality of the structure was calculated by ProSA web server [11]. It reads the atomic coordinates of the model and generates the Z-Score that is a determinant of the quality of the model.

Identification and alignment of kinase domains of Hexokinases The kinase domains of all HKs were identified by scanning their protein sequences against PROSITE data base that consists of documentation entries describing protein domains, families and functional sites as well as associated patterns and profiles [12]. The identified domains were aligned by multiple sequence alignment process using ClustalX tool to find out the similarities and dissimilarities among the domains.

\section{Superimposition of kinase domains}

The kinase domains of all HK structures were superimposed to find out the conformational variations using multiple 3D alignment module of MATRAS tool [13]. 
In HK-II only the $\gamma$-phosphate of ATP is found to be interacting with kinase domain along with its 2'-OH group of ribose sugar. The three phosphate groups and amino group of Adenine ring of ATP were found to be interacting with HK-III kinase domain (Figure 6). The docking scores for the four HKs indicated that HK-IV is showing best lowest docking score of -13.1834 and the remaining three $\mathrm{HKs}$ are showing almost similar docking scores of -12.417, -12.2306, -12.6445 for HK-I, HK-II and HK-III respectively Table 2 (see supplementary material). These values indicate the ATP-kinase domains complex strengths where the lowest value indicates the greater stability.

\section{Discussion:}

HKs work continuously to provide energy and thereby keeping the cells alive although they are tissue specific and confined to specific organs of the body. Their activity differs based on the requirement of energy sources at different stages [15]. Among the four HKs, the first three shows a low Km and HK-IV shows high $\mathrm{Km}$ and hence it is called as a predominant HK among HK family i.e. Glucokinase. This $\mathrm{Km}$ values determines the binding ability of the enzyme with substrate or ATP. This variation in the $\mathrm{Km}$ will result in the variable catalytic mechanism among their kinase domains [16]. This $\mathrm{Km}$ variation may be due to many factors and here in the present study we aimed to explain an important reason with respect to the binding affinities and orientation of ATP molecule in the binding site. Glucokinase which is HK-IV shows high affinity for glucose than other HKs and is the major enzyme associated with glucose phosphorylation in the body to process them to successive metabolic processes. Such a high phosphorylation rates may be due to high affinity towards substrate and ATP by its kinase domain [17]. Although the similar domains are shown by other HKs they are not showing high affinity as to that of HK-IV. The kinase domains of all HKs showed the similar conformation with two anti parallel $\beta$-sheets that providing the stability of the domains during catalysis. The structural alignment of kinase domains cleared that there is no conformational variation among them and the catalytic variation may not be due to the conformational variation of kinase domains (Figure 5). The residual composition of the domains is also found to be similar to major extent (Figure 4). The molecular docking study revealed the variation in the interaction of ATP which may explain the reasons for variable phosphorylation rates among four kinases. Interestingly it was found that the phosphate moiety of the ATP was in free form without interacting with the kinase domain residues in the HK-IV and hence it may be readily available for the phosphorylation of the sugars. But, in HK-I and HK-III, the $\alpha, \beta$, and $\gamma$ phosphates of the ATP were found to be in bound condition with their kinase domain residues, where as in HK-II, only $\gamma$ phosphate is found to be in bound condition to its kinase domain (Figure 6). In all these hexokinases, during catalytic process only $\gamma$ phosphate that is at free end will be donated to the $\mathrm{OH}$ group of the sixth carbon of hexose sugar [1]. In this study it was found that among first three HKs there is no availability of the free $\gamma$ phosphate where as it is available in HK-IV so that facilitating its transfer to hexose sugars. This might be an important evidence for the high $\mathrm{Km}$ and rapid phosphorylation rates by HK-IV than remaining HKs. This is more strengthened by their docking score variations where HK-IV showed a lowest docking score among all indicating the highest affinity for ATP than other HKs Table 2 (see supplementary material). Finally this study gave an insight into the variation of binding modes of ATP in the respective kinase domains. These orientations along with their variable docking scores may explain the variation and provide a theoretical basis for the variable phosphorylation rates among hexokinase family.

\section{Conclusion:}

The molecular docking of ATP into the kinase domains of four HKs revealed the binding mode variations and the phosphate moiety of ATP is found to be available only in HK-IV but not in remaining HKs. This might be responsible for their variable catalytic rates of phosphorylation.

\section{Acknowledgement:}

We are highly thankful for DST INSPIRE Division, Department of Science and Technology (DST), Government of India, for supporting with INSPIRE Fellowship.

\section{References:}

[1] Gonzalez C et al. Biochem Biophys Res Commun 1964 16: 347 [PMID: 5871820]

[2] Katzen HM \& Schimke RT, Proc Natl Acad Sci U S A. 1965 54: 1218 [PMID: 5219826]

[3] Wilson JE. J Exp Biol. 2003 206: 2049 [PMID: 12756287]

[4] http://www.proteopedia.org/wiki/index.php/Hexokinas e.

[5] Berman HM et al. Nucleic Acids Res. 2000 28: 235 [PMID: 10592235]

[6] Eswar $\mathrm{N}$ et al. Curr Protoc Bioinformatics. 2006 5: 5.[PMID: 18428767]

[7] http://www.ncbi.nlm.nih.gov/.

[8] Altschul SF et al. Nucleic Acids Res. 1997 25: 3389 [PMID: 9254694]

[9] Thompson JD et al. Nucleic Acids Res. 1997 25: 4876 [PMID: 9396791]

[10] Laskowski RA et al. J. Appl. Cryst. 1993 26: 283.

[11] Wiederstein M \& Sippl MJ, Nucleic Acids Res. 2007 35: W407 [PMID: 17517781]

[12] De Castro E et al. Nucleic Acids Res. 2006 34: W362 [PMID: 16845026]

[13] Kawabata T, Nucleic Acids Res. 2003 31: 3367 [PMID: 12824329]

[14] Wang $Y$ et al. Nucleic Acids Res. 2009 37: 623 [PMID: 19498078]

[15] Kanno H, Baillieres Best Pract Res Clin Haematol. 2000 13: 83 [PMID: 10916679]

[16] Grossbard L et al. J Biol Chem. 1966 241: 3546 [PMID: 5919684]

[17] Kawai S et al. J Biosci Bioeng. 2005 99: 320 [PMID: 16233797]

Edited by $P$ Kangueane

Citation: Kumar et al. Bioinformation 8(12): 543-547 (2012)

License statement: This is an open-access article, which permits unrestricted use, distribution, and reproduction in any medium, for non-commercial purposes, provided the original author and source are credited 


\section{BIOINFORMATION}

\section{Supplementary material:}

Table 1: RMSD matrix showing the values generated from super imposition of kinase domains of HKs by MATRAS tool. The values are the determinants of identity among kinase domains of four HKs.

\begin{tabular}{lllll}
\hline HK1 & 0.000 & 0.588 & 0.674 & 0.650 \\
HK2 & 0.588 & 0.000 & 0.233 & 0.520 \\
HK3 & $\mathbf{0 . 6 7 4}$ & $\mathbf{0 . 2 3 3}$ & $\mathbf{0 . 0 0 0}$ & $\mathbf{0 . 5 6 1}$ \\
HK4 & $\mathbf{0 . 6 5 0}$ & $\mathbf{0 . 5 2 0}$ & $\mathbf{0 . 5 6 1}$ & $\mathbf{0 . 0 0 0}$ \\
\hline
\end{tabular}

Table 2: Molecular docking of ATP into the kinase domains of HKs

\begin{tabular}{|c|c|c|c|c|}
\hline Hexokinase & Docking score & No. of H-Bonds & Interacting residues & H-bond length $(\AA)$ \\
\hline \multirow[t]{6}{*}{ HK1 } & -12.417 & 6 & G170 & 2.6 \\
\hline & & & K621 & 3.0 \\
\hline & & & S682 & 2.8 \\
\hline & & & S682 & 2.8 \\
\hline & & & S682 & 2.9 \\
\hline & & & E742 & 2.9 \\
\hline \multirow[t]{3}{*}{ HK2 } & -12.2306 & 3 & H504 & 2.4 \\
\hline & & & Q608 & 2.8 \\
\hline & & & N609 & 2.5 \\
\hline \multirow[t]{5}{*}{ HK3 } & -12.6445 & 5 & T626 & 2.2 \\
\hline & & & R520 & 3.0 \\
\hline & & & L623 & 2.7 \\
\hline & & & N624 & 2.6 \\
\hline & & & N624 & 2.9 \\
\hline \multirow[t]{2}{*}{ HK4 } & -13.1834 & 2 & H156 & 1.8 \\
\hline & & & E157 & 2.5 \\
\hline
\end{tabular}

